Loading

The Journal of the Canadian Game Studies Association

\title{
Mental Health in 3D
}

\section{A Dimensional Model of Mental Illness Representation in Digital Games}

\section{Kelli Dunlap and Rachel Kowert}

Volume 14, Number 24, Fall 2021

URI: https://id.erudit.org/iderudit/1084842ar

DOI: https://doi.org/10.7202/1084842ar

See table of contents

Publisher(s)

Canadian Game Studies Association

ISSN

1923-2691 (digital)

Explore this journal

Cite this article

Dunlap, K. \& Kowert, R. (2021). Mental Health in 3D: A Dimensional Model of Mental Illness Representation in Digital Games. Loading, 14(24), 122-133.

https://doi.org/10.7202/1084842ar
Article abstract

There is a wealth of research on the depiction and impact of mental health representations in traditional media; however, less is known about video games. As the dominant form of media in the 21st century, video games uniquely portray mental illness in traditional ways as well as in ways unique to video games, such as in-game mechanics (e.g., sanity meters) and player-driven decision making. This paper outlines the importance of cultural messages relating to mental illness as conveyed through video games in terms of content and influence and presents a multi-dimensional model of analysis for the representation of mental illness in digital games. The aim of this paper is to provide a foundation for understanding how mental illness is represented in digital games, provide a new perspective for thinking critically about representation of mental illness in games, and overview a new framework for assessing video game content in this area.
Copyright (c) Kelli Dunlap, Rachel Kowert, 2021

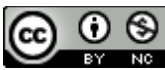

This document is protected by copyright law. Use of the services of Érudit (including reproduction) is subject to its terms and conditions, which can be viewed online.

https://apropos.erudit.org/en/users/policy-on-use/ 
Loading... The Journal of the Canadian Game Studies Association

Vol 14(24): 122-133

http://loading.gamestudies.ca

\title{
Mental Health in 3D: A Dimensional Model of Mental Illness Representation in Digital Games
}

\author{
Kelli Dunlap and Rachel Kowert \\ Dunlappsyd@gmail.com and rachel@takethis.org
}

\begin{abstract}
There is a wealth of research on the depiction and impact of mental health representations in traditional media; however, less is known about video games. As the dominant form of media in the 21 st century, video games uniquely portray mental illness in traditional ways as well as in ways unique to video games, such as in-game mechanics (e.g., sanity meters) and player-driven decision making. This paper outlines the importance of cultural messages relating to mental illness as conveyed through video games in terms of content and influence and presents a multidimensional model of analysis for the representation of mental illness in digital games. The aim of this paper is to provide a foundation for understanding how mental illness is represented in digital games, provide a new perspective for thinking critically about representation of mental illness in games, and overview a new framework for assessing video game content in this area.
\end{abstract}

\section{Author Keywords}

Mental illness, digital games

\section{Introduction}

Digital games are the dominant cultural form of the 21st century (Johnson, 2019). Recent reports from the Entertainment Software Association (2019) indicate $65 \%$ of American adults play video games and average about four hours a week of gameplay. The percentage of players under the age of 18 playing games is even greater and has been reported as high as $97 \%$ (Perrin, 2018). Video games continue to grow in popularity beyond the television, film, and music industries combined (Johnson, 2019).

Like traditional forms of storytelling, games convey the values and beliefs of the people and societies that create them. Even seemingly simple games like Pac-Man (Namco, 1980) or Mario Bros. (Nintendo, 1983) are digital records fossilized by play and convey a wealth of cultural information. For example, video game culture critic Jamin Warren explored the existential nature inherent in the design of PacMan, which is reflective of the time it was created and by the culture that created it: "PacMan is ultimately about the illusion of control. The reason we're chased by ghosts is that they are something from beyond that we can only pretend to have power over." (Warren, 2015).

Digital games have also rapidly developed into spaces for understanding, exploring, educating, and telling stories about mental health and mental illness. It is noteworthy that video games are a unique medium with specific affordances that differentiate them from other forms of media (Madigan, 2015). Thus, depictions of mental illness in gaming spaces are not just portrayed 
through game characters, settings, and stories but also through a game's mechanics (e.g., sanity meters) and players' in-game decisions.

The creation of digital games is the result of intentional and pain-staking design, and it's this design process that is the key to understanding current cultural values as well as creating cultural shifts. In other words, as digital games require every aspect of the play space to be coded, critically evaluating the design decisions provides insight into the underlying values, beliefs, and worldview of the designer and the consumer market they are catering to. These multidimensional media messages are particularly important to consider when discussing the ways in which mental health and illness are culturally valued and portrayed. Several researchers in the field have highlighted the importance of looking beyond depictions as being simply incorrect or harmful and instead called for a multi-faceted approach to assessing representation in games (Anderson, 2020; Dunlap, 2018; Stuart, 2006). However, no systematic approaches to doing so have yet to be developed. This is the primary aim of the current paper.

In order to understand the impact that mental health representation can have on our thoughts and behaviors relating to mental health, we first need to unpack the ways in which our cultural beliefs are shaped by the media and the ways in which video games portray mental health.

\section{Influence of media messages}

Our cultural values take cues from the media that we consume (Lule, 2016). That is, how the media portrays different groups of people conveys a sense of cultural value (Klein \& Shiffman, 2009) that we then absorb and integrate into our collective thoughts, values, and beliefs (for a review, see Morgan \& Shanahan, 2010). This can have a positive impact; for instance, when individuals watch inspiring media their worldview has the potential to shift to a "kind world" perspective and incorporate more positive views of humanity (Oliver, Ash, \& Woolley, 2012). However, media messages can also have a more negative impact through the creation and perpetuation of stereotypes.

Stereotypes are shared cultural beliefs about a particular group of individuals regarding the relationship between one's group membership and a psychological attribute (Hamilton \& Rose, 1980; Judd \& Park, 1993). It is important to note that a stereotype is not simply a social categorization but creates bias in the ways in which one processes information about any particular group. That is, even when an individual is met with information that contradicts their stereotypical belief, they may continue to maintain it. While stereotypes in and of themselves are not necessarily malicious, the labeling and categorizing that coincides with them often contributes to discrimination (the unjust treatment of different categories of people or things) and marginalization (a separation between $u s$ and them), both of which have far-reaching, real-world consequences.

Research has found media messages to contribute to the creation and perpetuation of stereotypes for a range of topics, including body image (Herbozo, Tantleff-Dunn, Gokee-Larose, \& Thompson, 2004), race and ethnicity (Scharrer \& Ramasubramanian, 2015), gender roles and identity (Eccles, 1987; Gauntlett, 2003), and mental illness. In particular, the stigma that surrounds mental illness is largely generated and shaped by labeling and stereotyping, which 
very often comes from media messages (Adler, A. K., \& Wahl, 1998; Ma, 2017; Stuart, 2006; Weiss, 1986, 1994).

Mass media has been found to be the most common public source of information about mental illness, and media portrayals of mental illness tend to be negative, exaggerated, and inaccurate (Ma, 2017; Singorielli, 1989; Stout, Villegas, \& Jennings, 2004; Wahl, 1995), with individuals with mental illness often depicted as violent, criminal, dangerous, and psychopathic (Stuart, 2006). Some of the typical mental health tropes used in film and television include the homicidal maniac, the rebellious free spirit, the specially gifted or enlightened oddball, the female patientseductress, the narcissistic parasite, the zoo specimen, the simpleton, and the failure or victim (Hyler, 2003; Pirkis, Blood, Francis, \& McCallum, 2005).

Previous research has found that exposure to negative media portrayals of mental illness are linked to negative and stereotyped perceptions of the mentally ill (Ma, 2017; McGinty, Webster, \& Barry, 2013; Stout et al., 2004) and to a reduction in help-seeking behaviors for individuals with mental illness (Stuart, 2006). Diefenbach and West (2007) found that heavy viewers of television (i.e., those exposed to high levels of media messaging) were more likely to believe that mental health services in residential neighborhoods pose a danger to the residents of those neighborhoods. Similarly, Granello and Pauley (2000) found that heavy viewers expressed less tolerance regarding mental illness. Stereotypical beliefs about mental illness have been also linked to social discrimination in job and housing opportunities (Link, Struening, Rahav, Phelan, \& Nuttbrock, 1997; Stuber, Meyer, \& Link, 2008; Wahl, 1999).

\section{Mental health portrayal in digital games}

While the portrayal of mental illness has been well-researched in television and film, research examining the ways mental health is portrayed in video games has been limited.

Morris and Forrest (2013) evaluated mental illness representation in a single video game, Batman: Arkham Asylum (Rocksteady Studios, 2009). In this action-adventure game, Batman battles his way out of an asylum for the criminally insane after being trapped inside. The authors summarized the portrayals of the mentally ill as featuring "stereotypical and discriminatory mental health representations commonly found in other video games" (pp. 757-758). Asylum inmates were depicted as "feral" and "animal-like" and were referred to by guards as "psychos," "insane," and "freaks." The Joker is even directly referred to in the game as a violent "schizophrenic," a clinical term for persons experiencing a constellation of symptoms such as hallucinations, delusions, lack of emotional expression, and disorganized speech or behavior (American Psychiatric Association, 2013).

An editorial article by games critic Patrick Lindsey (2014), titled "Gaming's favorite villain is mental illness, and this needs to stop", discussed the tendency of games to depict mentally ill characters as broken, less than human, and completely foreign. According to Lindsey, "These characters are dehumanized, portrayed as mental disorders embodied and wrapped in ostensibly human packaging." In instances when the mentally ill are more than subhuman entities, they tend to be murderous villains. Lindsey also called out the use of "sanity meters," a graphical user interface that quantifies how "crazy" a character is, as an example of mental illness as a game mechanic. 
In his editorial piece for Kotaku entitled "Nobody Wins When Horror Games Stigmatize Mental Illness", neuroscientist Ian Mahr (2013) dove into the common tropes associated with mental illness in horror games. These tropes related to the "crazed killer" and "horrific insane asylum" ones were identified as common story devices used to tie up loose exposition or backstory, or to provide justification for a character's behavior. His analysis reflected established tropes in media around the mentally ill as dangerous and particularly violent, and also emphasized the role psychiatric environments and artifacts (i.e., straitjackets, medication, etc.) play in portraying mental illness in games.

Shapiro and Rotter (2016) mapped the typical mental health tropes used in film and television (e.g., homicidal maniac, seductress, the savant) in top selling video games between 2011 and 2013 , and found that $24 \%$ depicted one or more mentally ill characters. Of the 42 individual characters that were identified as portraying mental illness, 29 (69\%) acted violently and in line with the homicidal maniac trope. Other researchers have found this trope to be particularly prevalent in gaming spaces (Ferrari, Bush, Clark, \& Archie, 2016; Morris \& Forrest, 2013). The use of this trope in media is particularly troublesome as one-in-three people report being fearful of persons with a mental illness (American Psychological Association, 2019), while the reality is that individuals with mental illness are more likely to be a victim of violence than persons without a mental illness (MentalHealth.gov, 2017).

Adopting mental illness tropes from television or film is a natural starting point for understanding representation in games; however, using this frame has resulted in a nearuniversal finding of negative and stereotyped portrayals and does not further future research into the ways in which mental illness is represented in these spaces. Similarly, it does not provide game designers any helpful feedback or guidance on how to create portrayals of mental illness that are anything other than exaggerated, harmful, or stereotyped. As video games are a unique medium with specific affordances that differentiate them from other forms of media (Madigan, 2015), the frames used to identify and analyze mental illness in games must go beyond those already established for television and film.

Anderson (2020) provides the first known attempt to address this multi-dimensionality by taking a play-centric approach to assessing mental health representation in games to assess the ways mental illness is portrayed in games. Through an assessment of three different games, Anderson found mental health is often portrayed beyond simple tropes, extending in ways specific to video games as a medium, including audiovisual styles, control systems, game goals, and procedurality.

While knowing what portrayals of mental illness are present in video games is valuable, we still do not have a full understanding of how mental illness is portrayed in games. This is because the content of our media "mediate[s] between existence and consciousness of existence, and thereby contributes to both" (Gerbner, 1990, p. 251). That is, the messages and images that surround us both reflect and produce the way we think about the world. As the content of our media conveys a sense of cultural value by reflecting our attitudes, beliefs, and priorities (Klein \& Shiffman, 2009), our cultural values can shift based on the messages in the media we consume (Lule, 2016). These effects can range from cultivating new thoughts and ideas to reinforcing stereotypes (Shrum, 1995). Cultural level, mass-produced messages (like those in the media) "provide many of the raw materials of our consciousness (and of the terms of our perceptions)" (Gerbner, 1963, p. 39). As media content holds the potential to shape the way we perceive and 
interpret the world, by shifting an individual's thoughts and behaviors or shifting cultural attitudes, it is important to analyze and critique the content of these messages, particularly when it comes to mental health.

To understand how mental illness is represented in video games, a new framework must be developed to capture character portrayal of mental illness as well as game narratives, mechanics, and environments. As aforementioned, several researchers in the field have highlighted the importance of looking beyond cataloguing the ways mass media depict illness either incorrectly or harmfully and instead called for a multi-faceted approach to assessing representation in games (Anderson, 2020; Dunlap, 2018; Stuart, 2006). As such, this framework must include space for considering more progressive (e.g., Celeste; Matt Makes Games, 2018) and nuanced (e.g. Stardew Valley; ConcernedApe, 2016) portrayals of mental illness.

For example, both Final Fantasy VI (Square, 1994) and Depression Quest (Quinn, 2013) portray mental illness, but the latter explores what it means to live with mental illness whilst the former uses it as a convenient plot point. Similarly, Arkham Asylum from Batman and Ospedale Psichiatrico di Volterra from Town of Light (LKA, 2016) both feature psychiatric hospitals. However, the former is a caricatured, comic-book style rendition of what someone might imagine an inhumane psychiatric hospital to be whilst the latter is a psychiatric hospital modeled from 19th century Italy littered with historically accurate artifacts. These games have widely different goals but they both offer representations of psychopathology worthy of study.

\section{A 3D dimensional model for categorizing mental illness in digital games}

Identifying and analyzing mental illness representations in games is uniquely challenging as mental illness is not always outwardly visible. Even if it was, as noted above it is not sufficient to simply identify the presence of absence of the portrayal of mental illness in a game, as this kind of classification does not allow for any analysis of the depth or accuracy of that portrayal. For example, both Final Fantasy VI and Depression Quest portray mental illness, however the former uses it as a convenient plot point whilst the latter explores what it is like for individuals living with mental illness. Similarly, Batman and Town of Light are both set in mental rehabilitation facilities, but the former is a fictional locale reliant on tropes and stereotypes whilst the latter is modeled after a still-standing asylum from the 19th century full of historically accurate artifacts. These games have widely different goals and approaches but both offer representations of psychopathology worthy of study.

To overcome these challenges, we propose conceptualizing the representations of mental illness in digital games on a three-dimensional spectrum, as summarized in Figure 1.

Figure 1. Three dimensions of mental health in digital games 


\section{Mental Health Representation}

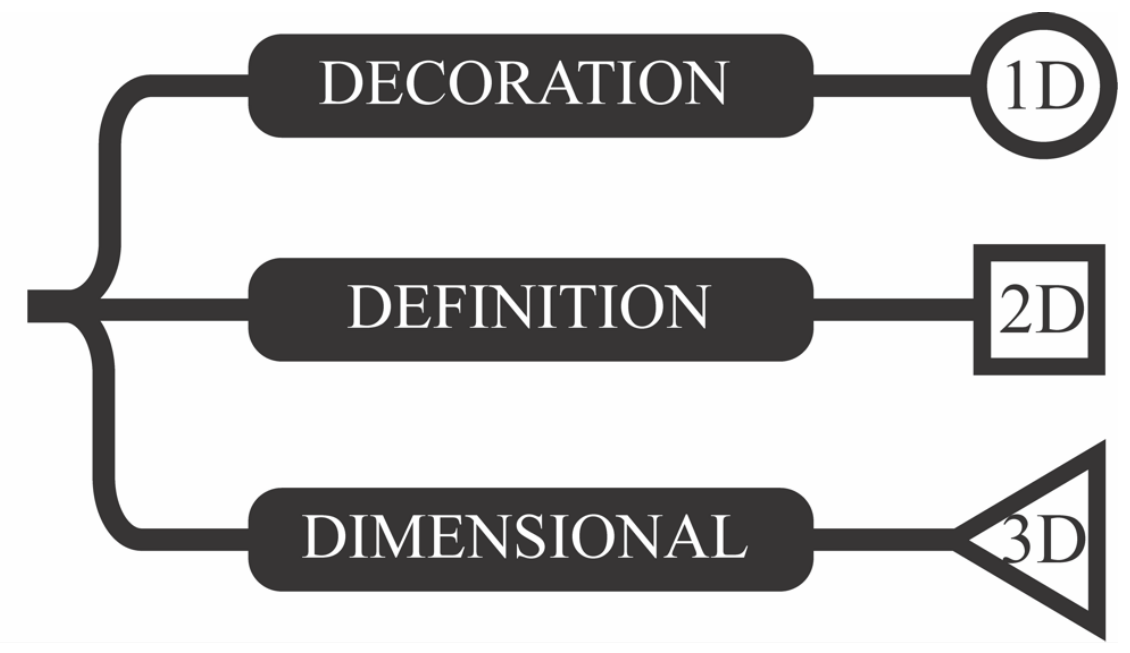

One-dimensional portrayals are flat and lack defining characteristics that would make them interesting (e.g., a straight line on a page) and not critical to the narrative of the game itself (i.e., they are purely "decorative"). Two-dimensional portrayals have specific "defining" properties that are integral to making them what they are (e.g., a square must have four points and removing or adding a point means it is no longer a square), but there is no nuance or depth to the representation. Three-dimensional portrayals extend beyond these specific properties and provide depth, dimensionality, and alternative perspectives to the representations (e.g., a cube).

Viewing mental illness portrayals on a spectrum such as this builds from the standards set by the American Psychiatric Association (2013) for assessing and diagnosing specific psychological disorders, that use a spectrum rather than the simple absence or presence of specific characteristics (e.g., autism spectrum disorder). Understanding mental health representations on a spectrum allows for the classification based on the types of representation found in media, rather than limiting all representations into distinct categories: good or bad; present or not; homicidal maniac or zoo specimen. Furthermore, a framework that allows for a spectrum of representations to exist allows for the classification and categorization of mental health representation to no longer be shoe-horned into a dichotomous box.

It is important to note that this classification model was not developed to diagnose characters but rather provide a more nuanced way to understand the representation of mental health in game characters, settings, and mechanics, by creating a framework robust enough to consider all portrayals, from the "decorative" (e.g., a random straight jacket in the corner of a room) to the multidimensional (e.g., fully developed characters and settings).

Each of the dimensions of the 3D model are discussed in more detail below.

\section{Decorative Representation (1D)}

The most simplistic representations of mental illness are one-dimensional representations. These consist of broad references to mental illness, where the representation is a non-essential element of a character, story, or environment. In other words, portrayals, descriptions, or depictions of mental health are decorative and carry minimal significance. 
For example, in The Elder Scrolls V: Skyrim (Bethesda Game Studios, 2013), there is a priest named Heimskr who gives loud sermons about the end of the world. The non-playable characters in the game (NPCs) often refer to him as mentally ill: "That Heimskr's crazy." There is no further attempt to understand Heimskr's motivations or behaviors. Labeling Heimskr as "crazy" is used to add flavor to the world, like a vendor selling sweet rolls or a guard talking about an arrow-induced knee pain. Removing the pejorative reference to his perceived level of sanity would not impact the game in any measurable way. Another example of a one-dimensional representation is the "illness in name" characters whose names presuppose the presence of a mental illness (Shapiro \& Rotter, 2016). For example, the Psychos from Borderlands (Gearbox Software, 2009), Crazy Red from Animal Crossing: New Leaf (Nintendo, 2012), and the Disturbed Suspect from L.A. Noire (Team Bondi, 2011). Another example from Overwatch (Blizzard Entertainment) is Sigma, a new character released in 2019. In an interview, an artist for the character noted that Sigma was intentionally designed barefooted to 'sell the asylum look' that contributed to his backstory of an escaped patient from a mental institution (Hernandez, 2019). These design choices do not impact story or character development and could be removed without impacting the character, narrative, or game setting.

\section{Defining Representation (2D)}

Two-dimensional representations are those whose psychopathological features are essential to, or a defining element of a character, story, or setting. The key element of these representations is that they lack depth. That is, the game does not explore mental illness beyond the surface level.

Two-dimensional representations often reflect commonly held views or beliefs about mental illness and serve as a kind of shorthand between the game developer and the player, enabling the developer to convey a wealth of information with minimal effort. For example, if a player wakes up in a psychiatric hospital it is unlikely that was due to a series of positive and fortunate events. Furthermore, it is unlikely that positive and fortunate events are to follow. The stereotype of a psychiatric hospital as a terrifying place where terrible things happen is pervasive, and in this instance serves as a convenient way to tell players to be afraid and expect trouble. Twodimensional representations are also those used to explain a character's behavior or backstory (for example, in Final Fantasy VI, Kefka, the game's antagonist, is motivated to destroy the world because he is "insane"). Such characteristics are important to defining these characters' identities and personalities but are not explored further.

\section{Dimensional Representation (3D)}

Three-dimensional representations of mental illness are fully realized and essential components of a character, narrative, or environment. These representations have depth and dimensionality, and examine the experience of mental illness from multiple perspectives. Three-dimensional representations reflect authentic experiences but do not necessarily need to be true-to-life in terms of design.

One example of a three-dimensional representation is Hellblade: Senua's Sacrifice (Ninja Theory, 2017). Hellblade features character, narrative, and environmental representations of mental illness (notably, schizophrenia) while also delivering a thoughtful and emotionally engaging story. For Senua, the game's protagonist, her mental illness influences how she moves 
through the world, but it does not solely define her. The audio design was crafted to simulate auditory hallucinations and implemented as a means to connect the player with Senua's experience, not for shock or terror (although they can be quite terrifying). The mechanics and environment of the game contribute to Senua's discomfort and reflect symptoms such as confusion, fear, and anxiety. The "rot" that creeps up Senua's arm after each failure and promises total game-progress erasure should it creep far enough is a prime example of a game's mechanics and narratives deeply engaging a player in the stakes of the game. By generating a shared sense of anxiety or dread, an empathic link between player and play-character is formed and the gameplay becomes a shared journey rather than a voyeuristic, mental-health-tourism romp through trauma.

A three-dimensional representation, however, does not require the massive resources used by Ninja Theory in creating Hellblade. For example, Neverending Nightmares (Ifinitap Games, 2014) is a black-and-white side-scrolling game with cartoon graphics and an evocative style. Although not realistic in terms of fidelity to the real world, the gameplay creates cognitive and emotional experiences that are multidimensional, complex, and reflect the lived experience of severe depression and obsessive-compulsive disorder as experienced by the game's creator.

A summary of these dimensions can be found in Table 1.

Table 1. Dimensional model of mental illness representation in games

\begin{tabular}{lccc}
\hline & One-Dimensional & Two-Dimensional & Three-Dimensional \\
\hline Key Feature & Decoration & Definition & Dimensional \\
Description & Broad reference & $\begin{array}{c}\text { Defining element with } \\
\text { limited or surface } \\
\text { representation }\end{array}$ & $\begin{array}{c}\text { Defining element with } \\
\text { depth and }\end{array}$ \\
& & dimensionality \\
Example & "Illness in name" & Kefka from Final & $\begin{array}{c}\text { Senua from Hellblade: } \\
\text { characters }\end{array}$ \\
& Fantasy VI & Senua's Sacrifice \\
\hline
\end{tabular}

It should be noted that one- and two-dimensional representations are not inherently problematic, as they can also provide neutral or even positive representations of mental illness. For example, one-dimensional portrayals could include an NPC mentioning in passing that they went to therapy, or a player could find a prescription for psychiatric medications or a psychiatrist's business card. Similarly, not all two-dimensional representations portray negative stereotypes. For example, Sandal from Dragon Age: Origins (Bioware, 2009) appears to have a cognitive impairment rendering him nearly mute. More noticeably, game developer Blizzard recently confirmed that the Overwatch character Symmetra is on the autism spectrum (Frank, 2017). That said, one- and two- dimensional representations have historically been found to reinforce and exaggerate stereotypes of mental illness rather than provide neutral or accurate representations. Without the context of a three-dimensional representation, one- and two-dimensional depictions generally lack the depth and dimensionality to provide accurate and insightful representation.

\section{Challenges and Limitations}


The 3D test provides a reasonably comprehensive framework to assess and evaluate mental health representation in games. However, we must note that the overwhelming amount of content in digital games remains a significant challenge for game developers and scholars. For example, a short, linear game like Gone Home (The Fullbright Company, 2013) takes around 2 hours to play through, whereas an open-world role-playing game like Red Dead Redemption (Rockstar Games, 2010) require dozens to hundreds of hours of gameplay to discover all its content. Compounding the problem is the sheer volume of games published each year: over 7,000 in 2018 alone (WePC, 2019). While an in-depth, well-played approach would be ideal, adopting a methodology that includes a specific selection of games, the use of game guides, and viewing videos of others who have played through the game (e.g., Shapiro \& Rotter, 2016) may be a necessary abstraction.

\section{Conclusion}

Portrayals of mental health and mental illness are common in video games yet there is little research available about what kind of representation is present. Video games are a complex medium and mental health is a complex topic; this combination renders traditional frameworks for studying and evaluating mental health inadequate when applied to games. A significant shortcoming of previous research on mental health representation in games is that existing paradigms (adapted from other visual mediums) are not able to capture the diversity and intensity with which mental health is represented in these games. The 3D model of mental health representation in games outlined in this paper is the first attempt to outline a foundation for researching, analyzing, and discussing the multifaceted ways in which mental health is portrayed in digital games.

The goals of this paper are to raise awareness about the casualness with which mental illness is often incorporated into gaming spaces, to lay the foundation for researchers to be able to consistently evaluate mental health portrayals in games, to view mental health representation as greater than only characters or narratives, and to encourage the design of multi-dimensional portrayals among game developers. We strongly encourage those conducting future work in this area to take a multi-faceted approach to understanding how in-game design choices speak to the cultural values and discourse around mental health and illness. The use of a dimensional model moves the discussion around mental health representation in games towards a more nuanced one and allows researchers and designers to consider a fuller range (or spectrum) of portrayals. As the largest form of popular media on the planet, researchers and designers have an obligation to better understand how media messages can shape the popular discourse in and around this important topic. 


\section{References}

Adler, A. K., \& Wahl, O. F. (1998). Children's beliefs about people labeled mentally ill. American Journal of Orthopsychiatry, 68(2), 321-326.

American Psychiatric Association. (2013). Diagnositc and statistical manual of mental disorders (5th ed.). Arlington, VA: American Psychiatric Publishing.

American Psychological Association. (2019). Survey: Americans becoming more open about mental health. Retrieved from https://www.apa.org/news/press/releases/apa-mental-healthreport.pdf

Anderson, S. L. (2020). Portraying mental illness in video games: Exploratory case studies for improving interactive depictions. Loading..., 13(21), 20-33.

Diefenbach, D. L., \& West, M. D. (2007). Television and attitudes toward mental health issues: Cultivation analysis and the third-person effect. Journal of Community Psychology, 35(2), $181-195$.

Dunlap, K. (2018). Representation of mental illness in video games. In 2018 Connected Learning Summit. Cambridge, MA: MIT Media Lab.

Eccles, J. S. (1987). Gender roles and women's achievement-related decisions. Psychology of Women Quarterly, 11(2), 135-172.

Entertainment Software Association. (2019). Essential facts about the computer and video game industry. Retrieved July 3, 2021, from https://www.theesa.com/wpcontent/uploads/2019/05/ESA_Essential_facts_2019_final.pdf

Ferrari, M., Bush, N., Clark, D., \& Archie, S. (2016). Debris: Exploring the video game values that can help reduce mental illness stigma. In 1st International Joint Conference of DiGRA and FDG.

Frank, A. (2017). Overwatch fan theory about Symmetra finally confirmed by Blizzard. Polygon. Retrieved from https://www.polygon.com/2017/3/9/14873308/overwatch-symmetra-autistic

Gauntlett, D. (2003). Media, gender and identity: An introduction. New York, NY: Routledge.

Gerbner, G. (1963). A theory of communication and its implications for teaching. In The Nature of Teaching (pp. 33-40). Milwaukee: University of Wisconsin-Milwaukee.

Gerbner, G. (1990). Epilogue: Advancing on the path of righteousness (maybe). In N. Signorielli \& M. Morgan (Eds.), Cultivation Analysis: New Directions in Media Effects Research (pp. 249-262). Newbury Park, CA: Sage.

Granello, D. H., \& Pauley, P. S. (2000). Television viewing habits and their relationship to tolerance toward people with mental illness. Journal of Mental Health Counseling, 22(2), $162-175$.

Hamilton, D. L., \& Rose, T. L. (1980). Illusory correlation and the maintenance of stereotypic beliefs. Journal of Personality and Social Psychology, 39(5), 832-845. 
Herbozo, S., Tantleff-Dunn, S., Gokee-Larose, J., \& Thompson, J. K. (2004). Beauty and thinness messages in children's media: A content analysis. Eating Disorders, 12(1), 21-34.

Hernandez, P. (2019). Overwatch artist says Sigma's bare feet meant to 'sell the asylum look'. Polygon.

Hyler, S. H. (2003). Stigma continues in Hollywood. Psychiatric Times, 20(6), 33-33.

Johnson, N. (2019). Report: Video games revenue was higher than Hollywood's in 2018.

Judd, C., \& Park, B. (1993). Definition and Assessment of Accuracy in Social Stereotypes. Psychological Review, 100(1), 109-128.

Klein, H., \& Shiffman, K. S. (2009). Underrepresentation and symbolic annihilation of socially disenfranchised groups ("out groups") in animated cartoons. The Howard Journal of Communication, 20(1), 55-72.

Lindsey, P. (2014). Gaming's favorite villian is mental illness and this needs to stop. Polygon. Retrieved from https:/www.polygon.com/2014/7/21/5923095/mental-health-gaming-silenthill

Link, B. G., Struening, E. L., Rahav, M., Phelan, J. C., \& Nuttbrock, L. (1997). On stigma and its consequences: evidence from a longitudinal study of men with dual diagnoses of mental illness and substance abuse. Ournal of Health and Social Behavior, 38(2), 177-190.

Lule, J. (2016). Understanding media and culture: An introduction to mass communication. Minneapolis, MN: University of Minnesota Library.

Ma, X. (2017). How the media covers mental illness: A review. Health Education, 117(11), 90109.

Madigan, J. (2015). Getting Gamers: The Psychology of Video Games and Their Impact on the People who Play. Lanham, MD: Rowman \& Littlefield Publishers.

Mahr, I. (2013). Nobody wins when horror games stigmatize mental illness. Kotaku2.

McGinty, E. E., Webster, D. W., \& Barry, C. L. (2013). Effects of news messages about mass shootings on attitudes towards persons with serious mental illness and public support for gun control policies. American Journal of Psychiatry, 170(5), 494-501.

MentalHealth.gov. (2017). Mental health myths and facts. Retrieved July 3, 2021, from https://www.mentalhealth.gov/basics/ mental-health-myths-facts

Morgan, M., \& Shanahan, J. (2010). The State of Cultivation. Journal of Broadcasting \& Electronic Media, 54(2), 337-355.

Morris, G., \& Forrest, R. (2013). Wham, sock, kapow! Can Batman defeat his biggest foe yet and combat mental health discrimination? An exploration of the video games industry and its potential for health promotion. Journal of Psychiatric and Mental Health Nursing, 20(8), $752-760$. 
Oliver, M. B., Ash, E., \& Woolley, J. K. (2012). The experience of elevation: Responses to media portrayals of moral beauty. In R. Tamborini (Ed.), Media and the moral mind (pp. 117-132). New York, NY: Routledge.

Perrin, A. (2018). Five facts about Americans and video games. Washington D.C. Retrieved from https://www.pewresearch.org/fact-tank/2018/09/17/ 5-facts-about-americans-andvideo-games $/ \% 0 \mathrm{~A}$

Pirkis, J., Blood, R. W., Francis, C., \& McCallum, K. (2005). A review of the literature regarding fictional film and television portrayals of mental illness. Melbourne, Australia.

Scharrer, E., \& Ramasubramanian, S. (2015). Intervening in the media's influence on stereotypes of race and ethnicity: The role of media literacy education. Journal of Social Issues, 71(1), $171-185$.

Shapiro, S., \& Rotter, M. (2016). Graphic depictions: Portrayals of mental illness in video games. Psychiatry and Behavioral Sciences, 61(6), 1592-1595.

Shrum, L. J. (1995). Shrum, L. J. (1995). Assessing the social influence of television: A social cognition perspective on cultivation effects. Communication Research, 22(4), 402-429.

Singorielli, N. (1989). The stigma of mental illness on television. Journal of Broadcasting \& Electronic Media, 33(3), 325-331.

Stout, P. A., Villegas, J., \& Jennings, N. A. (2004). Images of mental illness in the media: Identifying gaps in the research. Schizophrenia Bulletin, 30(3), 543-561.

Stuart, H. (2006). Media portrayal of mental illness and its treatments: What effect does it have on people with mental illness? CNS Drugs, 20(2), 99-106.

Stuber, J., Meyer, I., \& Link, B. (2008). Stigma, prejudice, discrimination and health. Social Science \& Medicine, 67(3), 351-357.

Wahl, O. F. (1995). Madness, madness everywhere. In Media madness: Public images of mental illness (pp. 1-13). New Brunswick, NJ: Rutgers University Press.

Wahl, O. F. (1999). Mental health consumers' experience of stigma. Schizophrenia Bulletin, 25(3), 467-478.

Warren, J. (2015). How Pac-Man's ghosts think and hunt. PBS Digital Studios. USA. Retrieved from https://youtu.be/xMEjovSlyqs

Weiss, M. F. (1986). Children's attitudes toward the mentally ill: A developmental analysis. Psychological Reports, 58(1), 11-20.

Weiss, M. F. (1994). Children's attitudes toward the mentally ill: an eight-year longitudinal follow-up. Psychological Reports, 74(1), 51-56.

WePC. (2019). 2019 Video game statistics, trends, and data. 